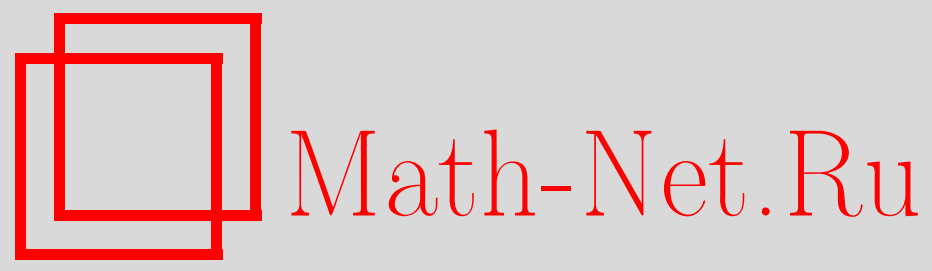

В. А. Емеличев, В. Г. Похилько, Анализ чувствительности эффективных решений векторной задачи минимизации линейных форм на множестве подстановок, Дискрет. матем., 2000, том 12, выпуск 3, 37-48

DOI: https://doi.org/10.4213/dm339

Использование Общероссийского математического портала Math-Net.Ru подразумевает, что вы прочитали и согласны с пользовательским соглашением http: //www.mathnet.ru/rus/agreement

Параметры загрузки:

IP : 34.229 .108 .108

26 апреля 2023 г., $13: 27: 20$

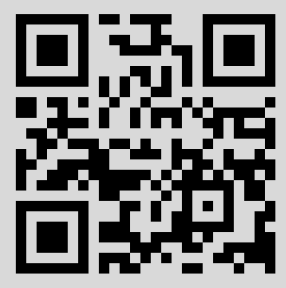




\title{
Анализ чувствительности эффективных решений векторной задачи минимизации линейных форм на множестве подстановок
}

\author{
(c) 2000 г. $\quad$ В. А. Емеличев, В. Г. Похилько
}

\begin{abstract}
Рассматривается многокритериальная постановка известной комбинаторной задачи минимизации линейной формы на произвольном множестве подстановок симметрической группы. Указаны границы изменений (в чебышевской метрике) коэффициентов линейных форм, сохраняющих соответствующую эффективность произвольного решения, оптимального по Парето, Слейтеру или Смейлу. Приводятся условия локальной устойчивости подстановки, обладающей свойством эффективности. Выделен класс квазиустойчивых задач.
\end{abstract}

\section{1. Определения и вспомогательные результаты}

Пусть $m, n \in \mathbf{N}, m \geqslant 2$, а $A=\left(a_{i j}\right)_{n \times m}$ и $B=\left(b_{i j}\right)_{n \times m}$ - пара вещественных матриц. Пусть $S_{m}$ - симметрическая группа подстановок, действующая на множестве $N_{m}=$ $\{1,2, \ldots, m\}$. На непустом множестве $T \subseteq S_{m}$ зададим векторный критерий

$$
f(t, A, B)=\left(f_{1}\left(t, A_{1}, B_{1}\right), f_{2}\left(t, A_{2}, B_{2}\right), \ldots, f_{n}\left(t, A_{n}, B_{n}\right)\right) \rightarrow \min _{t \in T}
$$

частными критериями которого являются функции

$$
f_{i}\left(t, A_{i}, B_{i}\right)=\sum_{j=1}^{m} a_{i j} b_{i t(j)}, \quad i \in N_{n},
$$

где $t=(t(1), \ldots, t(m))$.

Здесь и далее нижний индекс $i$ у матрицы (вектора) указывает на $i$-ю строку (компоненту) соответствующей матрицы (соответствующего вектора), например, $A_{i}=\left(a_{i 1}, a_{i 2}, \ldots, a_{i m}\right)$. В этом контексте традиционные определения (см., например, [1]) множества строго эффективных (оптимальных по Смейлу) подстановок, множества истинно эффективных (оптимальных по Парето) подстановок и множества слабо эффективных (оптимальных по Слейтеру) подстановок имеют соответственно вид

$$
T_{k}^{n}(A, B)=\left\{t \in T: \tau_{k}(t, A, B)=\varnothing\right\}, \quad k \in N_{3}
$$


где

$$
\begin{aligned}
\tau_{1}(t, A, B) & =\left\{t^{\prime} \in T \backslash\{t\}: q\left(t, t^{\prime}, A, B\right) \geqslant 0_{(n)}\right\}, \\
\tau_{2}(t, A, B) & =\left\{t^{\prime} \in T: q\left(t, t^{\prime}, A, B\right) \geqslant 0_{(n)}, q\left(t, t^{\prime}, A, B\right) \neq 0_{(n)}\right\}, \\
\tau_{3}(t, A, B) & =\left\{t^{\prime} \in T: \forall i \in N_{n}\left(q_{i}\left(t, t^{\prime}, A_{i}, B_{i}\right)>0\right)\right\}, \\
q\left(t, t^{\prime}, A, B\right) & =\left(q_{1}\left(t, t^{\prime}, A_{1}, B_{1}\right), \ldots, q_{n}\left(t, t^{\prime}, A_{n}, B_{n}\right)\right), \\
q_{i}\left(t, t^{\prime}, A_{i}, B_{i}\right) & =f_{i}\left(t, A_{i}, B_{i}\right)-f_{i}\left(t^{\prime}, A_{i}, B_{i}\right), \quad i \in N_{n}, \\
0_{(n)} & =(0, \ldots, 0) \in \mathbf{R}^{n} .
\end{aligned}
$$

Непосредственно из этих определений вытекают включения

$$
T_{1}^{n}(A, B) \subseteq T_{2}^{n}(A, B) \subseteq T_{3}^{n}(A, B) .
$$

Для всякого числа $k \in N_{3}$ задачу поиска множества эффективных подстановок $T_{k}^{n}(A, B)$ будем обозначать через $Z_{k}^{n}(A, B)$. Так как мы предположили непустоту множества $T$, то очевидно, что $T_{2}^{n}(A, B) \neq \varnothing$ и $T_{3}^{n}(A, B) \neq \varnothing$ при любых $A, B \in \mathbf{R}^{n m}$. Заметим, что множество строго эффективных подстановок $T_{1}^{n}(A, B)$ может быть и пустым. Однако, говоря в дальнейшем о задаче $Z_{1}^{n}(A, B)$, будем предполагать, что $T_{1}^{n}(A, B) \neq \varnothing$.

Очевидно, что при переходе к однокритериальному случаю $\left(n=1, A, B \in \mathbf{R}^{m}\right)$ множества $T_{2}^{1}(A, B)$ и $T_{3}^{1}(A, B)$ совпадут и превратятся во множество оптимальных подстановок, а наша задача - в широко известную скалярную задачу минимизации линейной формы на множестве подстановок (см., например, монографии [2, 3], а также обзор [4] и библиографию в нем). Очевидно, что в случае, когда оптимальная подстановка $t^{*}$ задачи $Z_{2}^{1}(A, B)$ единственна, справедливы равенства

$$
T_{1}^{1}(A, B)=T_{2}^{1}(A, B)=T_{3}^{1}(A, B)=\left\{t^{*}\right\} .
$$

В противном случае множество строго эффективных подстановок скалярной задачи пусто.

Известно, что одним из важных элементов решения практических задач оптимизации является проведение анализа чувствительности решения к изменениям параметров задачи. В данной статье будем изучать поведение эффективной подстановки при возмущениях элементов матрицы $A$. Возникает вопрос: насколько сильно можно изменять независимо друг от друга эти параметры, чтобы при этом выбранная эффективная подстановка в любой возмущенной задаче сохраняла соответствующую эффективность? Подобная количественная постановка проблемы устойчивости естественно приводит к ключевому понятию радиуса устойчивости, впервые введенному в $[5,6]$ для скалярных траекторных задач комбинаторной оптимизации.

Прежде, чем дать строгое определение радиуса устойчивости эффективной подстановки, введем некоторые новые понятия и сформулируем очевидные свойства.

Для числа $n \in \mathbf{N}$ и подстановки $t \in T_{k}^{n}(A, B), k=1,2$, введем множество

$$
W_{k}^{n}(t, A, B)=\left\{t^{\prime} \in T: I^{n}\left(t, t^{\prime}, B\right) \neq \varnothing\right\},
$$

где

$$
\begin{aligned}
I^{n}\left(t, t^{\prime}, B\right) & =\left\{i \in N_{n}: \delta_{i}\left(t, t^{\prime}, B_{i}\right)>0\right\} \\
\delta_{i}\left(t, t^{\prime}, B_{i}\right) & =\sum_{j=1}^{m}\left|b_{i t(j)}-b_{i t^{\prime}(j)}\right|
\end{aligned}
$$


При $t \in T_{3}^{n}(A, B)$ положим

$$
W_{3}^{n}(t, A, B)=\left\{t^{\prime} \in T: I^{n}\left(t, t^{\prime}, B\right)=N_{n}\right\} .
$$

Очевидно, что для всякой подстановки $t \in T_{1}^{n}(A, B)$ справедливо равенство

$$
W_{1}^{n}(t, A, B)=T \backslash\{t\},
$$

а также верны соотношения

$$
W_{1}^{n}(t, A, B)=W_{2}^{n}(t, A, B) \supseteq W_{3}^{n}(t, A, B),
$$

а для $t \in T_{2}^{n}(A, B)$ справедливо включение $W_{2}^{n}(t, A, B) \supseteq W_{3}^{n}(t, A, B)$.

Эффективную подстановку $t \in T_{k}^{n}(A, B)$ назовем нетривиальной, если множество $W_{k}^{n}(t, A, B)$ не пусто. В противном случае подстановку будем называть тривиальной. Множество всех нетривиальных подстановок из $T_{k}^{n}(A, B)$ будем обозначать через $\dot{T}_{k}^{n}(A, B)$. Ясно, что $\dot{T}_{1}^{n}(A, B)=T_{1}^{n}(A, B)$ при $|T|>1$. Тем самым, подстановка $t \in T_{1}^{n}(A, B)$ тривиальна лишь в случае, когда $|T|=1$. Кроме того, нетрудно понять, что в отношении множества $\dot{T}_{2}^{n}(A, B)$ возможны лишь два случая: либо $\dot{T}_{2}^{n}(A, B)=T_{2}^{n}(A, B)$, либо $\dot{T}_{2}^{n}(A, B)=\varnothing$. Тем самым, или все истинно эффективные подстановки задачи $Z_{2}^{n}(A, B)$ нетривиальны, или все они тривиальны. Относительно множества $\dot{T}_{3}^{n}(A, B)$ подобное утверждение, вообще говоря, неверно (см. ниже пример 3).

Как мы уже условились, возмущать будем лишь элементы матрицы $A$ путем сложения с соответствующими элементами матрицы $A^{\prime} \in \mathbf{R}^{n \times m}$. Для всякого натурального числа $d$ под нормой вектора $x=\left(x_{1}, \ldots, x_{d}\right) \in \mathbf{R}^{d}$ будем понимать норму в $l_{\infty}$ :

$$
\|x\|=\max \left\{\left|x_{i}\right|: i \in N_{d}\right\} .
$$

В приведенных выше обозначениях справедливы следующие очевидные свойства.

Свойство 1. Если справедлива формула

$$
\exists t^{\prime} \in T \forall i \in N_{n}\left(q_{i}\left(t, t^{\prime}, A_{i}, B_{i}\right)>0\right),
$$

то $t \notin T_{3}^{n}(A, B)$.

Свойство 2. Справедлива формула

$$
\forall k \in N_{3} \forall t \in \dot{T}_{k}^{n}(A, B) \forall t^{\prime} \in W_{k}^{n}(t, A, B)\left(I^{n}\left(t, t^{\prime}, B\right) \neq \varnothing\right) .
$$

Свойство 3. Если $I^{n}\left(t, t^{\prime}, B\right) \neq N_{n}$, то верна формула

$$
\forall i \in N_{n} \backslash I^{n}\left(t, t^{\prime}, B\right) \forall A_{i}^{\prime} \in \mathbf{R}^{m}\left(q_{i}\left(t, t^{\prime}, A_{i}+A_{i}^{\prime}, B_{i}\right)=0\right) .
$$

Свойство 4. Если различные подстановки $t, t^{\prime} \in T$, индекс $i \in N_{n}$ и вектор $A_{i}^{\prime} \in \mathbf{R}^{m}$ таковы, что

$$
q_{i}\left(t, t^{\prime}, A_{i}, B_{i}\right)+\left\|A_{i}^{\prime}\right\| \delta_{i}\left(t, t^{\prime}, B_{i}\right)<0,
$$

то имеет место неравенство

$$
q_{i}\left(t, t^{\prime}, A_{i}+A_{i}^{\prime}, B_{i}\right)<0 .
$$


Действительно, с учетом очевидного неравенства

$$
q_{i}\left(t, t^{\prime}, A_{i}^{\prime}, B_{i}\right) \leqslant\left\|A_{i}^{\prime}\right\| \delta_{i}\left(t, t^{\prime}, B_{i}\right)
$$

находим, что

$$
\begin{aligned}
q_{i}\left(t, t^{\prime}, A_{i}+A_{i}^{\prime}, B_{i}\right) & =q_{i}\left(t, t^{\prime}, A_{i}, B_{i}\right)+q_{i}\left(t, t^{\prime}, A_{i}^{\prime}, B_{i}\right) \\
& \leqslant q_{i}\left(t, t^{\prime}, A_{i}, B_{i}\right)+\left\|A_{i}^{\prime}\right\| \delta_{i}\left(t, t^{\prime}, B_{i}\right)<0 .
\end{aligned}
$$

Для всякого фиксированного числа $k \in N_{3}$ радиусом устойчивости подстановки $t \in T_{k}^{n}(A, B)$ называется величина

$$
\rho_{k}^{n}(t, A, B)= \begin{cases}\sup \Omega, & \text { если } \Omega \neq \varnothing, \\ 0 & \text { в противном случае }\end{cases}
$$

где

$$
\begin{aligned}
\Omega & =\left\{\varepsilon>0: \forall A^{\prime} \in \mathscr{A}(\varepsilon)\left(t \in T_{k}^{n}\left(A+A^{\prime}, B\right)\right)\right\}, \\
\mathscr{A}(\varepsilon) & =\left\{A^{\prime} \in \mathbf{R}^{n \times m}:\left\|A^{\prime}\right\|<\varepsilon\right\} .
\end{aligned}
$$

Здесь и далее под нормой матрицы понимается норма вектора, составленного из ее элементов.

Таким образом, радиус устойчивости эффективной подстановки задает предел независимых возмущений элементов матрицы $A$, при которых соответствующая эффективность подстановки сохраняется.

Ввиду включений (1) для любой подстановки $t \in T_{1}^{n}(A, B)$ выполняются неравенства

$$
\rho_{1}^{n}(t, A, B) \leqslant \rho_{2}^{n}(t, A, B) \leqslant \rho_{3}^{n}(t, A, B),
$$

а для всякой подстановки $t \in T_{2}^{n}(A, B)$ справедливо неравенство

$$
\rho_{2}^{n}(t, A, B) \leqslant \rho_{3}^{n}(t, A, B) .
$$

Задачу $Z_{k}^{n}\left(A+A^{\prime}, B\right)$, где $A^{\prime} \in \mathscr{A}(\varepsilon)$, полученную из исходной задачи $Z_{k}^{n}(A, B)$ путем сложения матриц $A$ и $A^{\prime}$, будем называть возмущенной, а матрицу $A^{\prime}$ возмущающей.

Естественно считать, что радиус устойчивости подстановки $t \in T_{k}^{n}(A, B)$ бесконечен, если $t \in T_{k}^{n}\left(A+A^{\prime}, B\right)$ для всех матриц $A^{\prime} \in \mathbf{R}^{n \times m}$.

Предложение 1. Радиус устойчивости всяхой тривиальной эффехтивной подстановки равен бесконечности.

Доказательство. Пусть подстановка $t \in T_{k}^{n}(A, B), k \in N_{3}$, тривиальна. Тогда множество $W_{k}^{n}(t, A, B)$ пусто. В случае, когда $k=1$, утверждение очевидно, поскольку, как уже отмечалось, $|T|=1$.

Далее пусть $k=2$. Тогда в соответствии с определением множества $W_{2}^{n}(t, A, B)$ для любой подстановки $t^{\prime} \in T$ множество $I^{n}\left(t, t^{\prime}, B\right)$ пусто. Поэтому благодаря свойству 3 справедлива формула

$$
\forall t^{\prime} \in T \forall i \in N_{n} \forall A_{i}^{\prime} \in \mathbf{R}^{m}\left(q_{i}\left(t, t^{\prime}, A_{i}+A_{i}^{\prime}, B_{i}\right)=0\right),
$$


которая и означает, что $t \in T_{2}^{n}\left(A+A^{\prime}, B\right)$ при любой матрице $A^{\prime} \in \mathbf{R}^{n \times m}$, то есть $\rho_{2}^{n}(t, A, B)=\infty$.

Наконец, пусть $k=3$. Тогда согласно определению множества $W_{3}^{n}(t, A, B)$ при любой подстановке $t^{\prime} \in T$ верно неравенство $I^{n}\left(t, t^{\prime}, B\right) \neq N_{n}$ и поэтому с учетом свойства 3 справедлива формула

$$
\forall t^{\prime} \in T \forall i \in N_{n} \backslash I^{n}\left(t, t^{\prime}, B\right) \forall A_{i}^{\prime} \in \mathbf{R}^{m}\left(q_{i}\left(t, t^{\prime}, A_{i}+A_{i}^{\prime}, B_{i}\right)=0\right) .
$$

Сказанное означает, что $t \in T_{3}^{n}\left(A+A^{\prime}, B\right)$ при любой матрице $A^{\prime} \in \mathbf{R}^{n \times m}$, то есть $\rho_{3}^{n}(t, A, B)=\infty$.

Предложение доказано.

В свете доказанного предложения вывод формулы для радиуса устойчивости проведем для нетривиальной эффективной подстановки. Непосредственно из определения радиуса устойчивости эффективной подстановки вытекают следующие два свойства.

Свойство 5. Пусть $t \in \dot{T}_{k}^{n}(A, B), k \in N_{3}$. Если существует такое число $\varphi>0$, что $t \in T_{k}^{n}\left(A+A^{\prime}, B\right)$ для всякой возмущающей матрицы $A^{\prime} \in \mathscr{A}(\varphi)$, то $\rho_{k}^{n}(t, A, B) \geqslant \varphi$.

Свойство 6. Пусть $t \in \dot{T}_{k}^{n}(A, B), \quad k \in N_{3}, \varphi \geqslant 0$ и для всякого числа $\varepsilon>\varphi$ найдется возмущающая матрица $A^{\prime} \in \mathscr{A}(\varepsilon)$, удовлетворяющая условию $t \notin$ $T_{k}^{n}\left(A+A^{\prime}, B\right)$. Тогда $\rho_{k}^{n}(t, A, B) \leqslant \varphi$.

Нам понадобится также следующая лемма.

Лемма 1. Пусть $t \in \dot{T}_{k}^{n}(A, B), k \in N_{3}, \varphi>0$ и справедлива формула

$$
\forall A^{\prime} \in \mathscr{A}(\varphi) \forall t^{\prime} \in W_{k}^{n}(t, A, B) \exists i \in I^{n}\left(t, t^{\prime}, B\right)\left(q_{i}\left(t, t^{\prime}, A_{i}+A_{i}^{\prime}, B_{i}\right)<0\right) .
$$

Тогда $t \in T_{k}^{n}\left(A+A^{\prime}, B\right)$ при любой матрице $A^{\prime} \in \mathscr{A}(\varphi)$.

Доказателъство. Доказательство проведем отдельно для каждого из значений числа $k$.

Пусть $k=1$. Тогда $W_{1}^{n}(t, A, B)=T \backslash\{t\} \neq \varnothing$. Поэтому (3) означает, что для всякой возмущающей матрицы $A^{\prime} \in \mathscr{A}(\varphi)$ множество $\tau_{1}\left(t, A+A^{\prime}, B\right)$ пусто, то есть $t \in T_{1}^{n}\left(A+A^{\prime}, B\right)$.

При $k=2$ пусть $t^{\prime} \notin W_{2}^{n}(t, A, B)$. Тогда $I^{n}\left(t, t^{\prime}, B\right)=\varnothing$. Поэтому согласно свойству 3 верны равенства

$$
q_{i}\left(t, t^{\prime}, A_{i}+A_{i}^{\prime}, B_{i}\right)=0, \quad i \in N_{n},
$$

для любой матрицы $A^{\prime} \in \mathbf{R}^{n \times m}$. Отсюда с учетом формулы (3) заключаем, что при любой матрице $A^{\prime} \in \mathscr{A}(\varphi)$ множество $\tau_{2}\left(t, A+A^{\prime}, B\right)=\varnothing$ и, следовательно, $t \in T_{2}^{n}\left(A+A^{\prime}, B\right)$.

Пусть $k=3$. Если $t^{\prime} \notin W_{3}^{n}(t, A, B)$, то $I^{n}\left(t, t^{\prime}, B\right) \neq N_{n}$. Таким образом, на основании свойства 3 для любого индекса $i \in N_{n} \backslash I^{n}\left(t, t^{\prime}, B\right)$ и любого вектора $A_{i}^{\prime} \in \mathbf{R}^{m}$ справедливо равенство

$$
q_{i}\left(t, t^{\prime}, A_{i}+A_{i}^{\prime}, B_{i}\right)=0 .
$$

Отсюда видно (ввиду (3)), что для всякой возмущающей матрицы $A^{\prime} \in \mathscr{A}(\varphi)$ множество $\tau_{3}\left(t, A+A^{\prime}, B\right)$ пусто, то есть $t \in T_{3}^{n}\left(A+A^{\prime}, B\right)$.

Лемма доказана. 


\section{2. Основной результат}

Для нетривиальной подстановки $t \in \dot{T}_{k}^{n}(A, B), k \in N_{3}$, введем обозначения

$$
\begin{aligned}
\varphi_{k}^{n}(t, A, B) & =\min _{t^{\prime} \in W_{k}^{n}(t, A, B)} \max _{i \in I^{n}\left(t, t^{\prime}, B\right)} \Gamma_{i}\left(t, t^{\prime}, A_{i}, B_{i}\right), \\
\Gamma_{i}\left(t, t^{\prime}, A_{i}, B_{i}\right) & =-\frac{q_{i}\left(t, t^{\prime}, A_{i}, B_{i}\right)}{\delta_{i}\left(t, t^{\prime}, B_{i}\right)} .
\end{aligned}
$$

Теорема 1. При любах $k \in N_{3}$ u $n \in \mathbf{N}$ для радиуса устойчивости вслхой нетривиальной эффективной подстановки $t \in \dot{T}_{k}^{n}(A, B)$ векторной задачи $Z_{k}^{n}(A, B)$ справедлива формула

$$
\rho_{k}^{n}(t, A, B)=\varphi_{k}^{n}(t, A, B)
$$

Доказательство. Пусть $t \in \dot{T}_{k}^{n}(A, B), k \in N_{3}$. Тогда $W_{k}^{n}(t, A, B) \neq \varnothing$. Из свойства 2 следует непустота множества $I^{n}\left(t, t^{\prime}, B\right)$ для любой подстановки $t^{\prime} \in W_{k}^{n}(t, A, B)$. Нетрудно видеть, что

$$
\varphi=\varphi_{k}^{n}(t, A, B) \geqslant 0 .
$$

Переходя к доказательству неравенства $\rho_{k}^{n}(t, A, B) \geqslant \varphi$, будем предполагать, что $\varphi>0$ (в случае, когда $\varphi=0$, это неравенство очевидно). Тогда множество возмущающих матриц $\mathscr{A}(\varphi) \neq \varnothing$, и согласно определению числа $\varphi$ для любой матрицы $A^{\prime} \in \mathscr{A}(\varphi)$ справедлива формула

$$
\forall t^{\prime} \in W_{k}^{n}(t, A, B) \exists i \in I^{n}\left(t, t^{\prime}, B\right)\left(\left\|A_{i}^{\prime}\right\|<\varphi \leqslant \Gamma_{i}\left(t, t^{\prime}, A_{i}, B_{i}\right)\right) .
$$

Откуда благодаря свойству 4 заключаем, что верно неравенство

$$
q_{i}\left(t, t^{\prime}, A_{i}+A_{i}^{\prime}, B_{i}\right)<0 .
$$

Поэтому, применяя лемму, убеждаемся, что подстановка $t \in T_{k}^{n}\left(A+A^{\prime}, B\right)$ при любой матрице $A^{\prime} \in \mathscr{A}(\varphi)$. Следовательно, в силу свойства $5 \rho_{k}^{n}(t, A, B) \geqslant \varphi$.

Теперь докажем неравенство $\rho_{k}^{n}(t, A, B) \leqslant \varphi$. Согласно определению числа $\varphi$ справедлива формула

$$
\exists t^{\prime} \in W_{k}^{n}(t, A, B) \forall i \in I^{n}\left(t, t^{\prime}, B\right)\left(\varphi \geqslant \Gamma_{i}\left(t, t^{\prime}, A_{i}, B_{i}\right)\right) .
$$

Заметим, что в силу свойства 2 множество $I^{n}\left(t, t^{\prime}, B\right) \neq \varnothing$. Пусть $\varepsilon>\varphi$. Рассмотрим возмущающую матрицу $A^{*}=\left(a_{i j}^{*}\right)_{n \times m}$, элементы которой для пар $(i, j) \in$ $N_{n} \times N_{m}$ задаются формулой

$$
a_{i j}^{*}= \begin{cases}\alpha & \text { при } b_{i t(j)} \geqslant b_{i t^{\prime}(j)} \\ \alpha & \text { при } b_{i t(j)}<b_{i t^{\prime}(j)}\end{cases}
$$

где $\varphi<\alpha<\varepsilon$. Очевидно, что матрица $A^{*} \in \mathscr{A}(\varepsilon)$.

С учетом неравенства (4) и структуры матрицы $A^{*}$ легко убедиться в справедливости для любого индекса $i \in I^{n}\left(t, t^{\prime}, B\right)$ следующих соотношений

$$
\begin{aligned}
q_{i}\left(t, t^{\prime}, A_{i}+A_{i}^{*}, B_{i}\right) & =q_{i}\left(t, t^{\prime}, A_{i}, B_{i}\right)+\alpha \delta_{i}\left(t, t^{\prime}, B_{i}\right) \\
& \geqslant(\alpha-\varphi) \delta_{i}\left(t, t^{\prime}, B_{i}\right)>0 .
\end{aligned}
$$


Далее рассмотрим два случая.

Пусть $I^{n}\left(t, t^{\prime}, B\right)=N_{n}$. Тогда, как легко видеть, в силу (5) и свойства 1

$$
\forall \varepsilon>\varphi \exists A^{*} \in \mathscr{A}(\varepsilon)\left(t \notin T_{3}^{n}\left(A+A^{*}, B\right)\right) .
$$

Поэтому из (1) получаем, что $t \notin T_{k}^{n}\left(A+A^{*}, B\right)$. Следовательно, согласно свойству 6 справедливо неравенство $\rho_{k}^{n}(t, A, B) \leqslant \varphi$.

Пусть теперь $I^{n}\left(t, t^{\prime}, B\right) \neq N_{n}$. По предположению подстановка $t \in \dot{T}_{k}^{n}(A, B)$, поэтому $W_{k}^{n}(t, A, B) \neq \varnothing$. Следовательно, в этом случае согласно определению множества $W_{3}^{n}(t, A, B)$ число $k \neq 3$. Итак, $t \in \dot{T}_{2}^{n}(A, B)$. Из свойства 3 выводим, что для любого индекса $i \in N_{n} \backslash I^{n}\left(t, t^{\prime}, B\right)$ верно равенство

$$
q_{i}\left(t, t^{\prime}, A_{i}+A_{i}^{*}, B_{i}\right)=0 .
$$

Поэтому с учетом (5) справедлива формула

$$
\forall \varepsilon>\varphi \exists A^{*} \in \mathscr{A}(\varepsilon)\left(t \notin T_{2}^{n}\left(A+A^{*}, B\right)\right) .
$$

Отсюда в силу свойства 6 и неравенств (2) получаем, что

$$
\rho_{1}^{n}(t, A, B) \leqslant \rho_{2}^{n}(t, A, B) \leqslant \varphi .
$$

Теорема доказана.

\section{3. Следствия}

Непосредственно из теоремы извлекаются следующие утверждения.

Следствие 1. Радиус устойчивости $\rho_{k}^{n}(t, A, B), n \in \mathrm{N}, k \in N_{3}$, нетривиалъной эффективной подстановки $t$ конечен.

Следствие 2. При $t \in T_{1}^{n}(A, B)$ справедливо равенство

$$
\rho_{1}^{n}(t, A, B)=\rho_{2}^{n}(t, A, B) .
$$

Следствие 3. Для любых $n \in \mathbf{N} u t \in \dot{T}_{2}^{n}(A, B)$ верна эквивалентность

$$
\rho_{2}^{n}(t, A, B)=0 \Longleftrightarrow \exists t^{\prime} \in W_{2}^{n}(t, A, B)\left(q\left(t, t^{\prime}, A, B\right)=0_{(n)}\right) .
$$

Следствие 4. Для любых $n \in \mathbf{N} u t \in \dot{T}_{3}^{n}(A, B)$ верна эквивалентность

$$
\rho_{3}^{n}(t, A, B)=0 \Longleftrightarrow \exists t^{\prime} \in W_{3}^{n}(t, A, B)\left(q\left(t, t^{\prime}, A, B\right) \geqslant 0_{(n)}\right) .
$$

При $n=1$ теорема переходит в следующее утверждение.

Следствие 5. Для радиуса устойчивости оптималъной подстановки $t^{*} \in T_{2}^{1}(A, B)$ скалярной задачи $Z_{2}^{1}(A, B)$

$$
A=\left(a_{1}, \ldots, a_{m}\right) \in \mathbf{R}^{m}, \quad B=\left(b_{1}, \ldots, b_{m}\right) \in \mathbf{R}^{m}, \quad m \geqslant 2,
$$

справедлива формула

$$
\rho_{2}^{1}\left(t^{*}, A, B\right)= \begin{cases}\Psi, & \text { eсли } t^{*} \in \dot{T}_{2}^{1}(A, B), \\ \infty, & \text { если } t^{*} \notin \dot{T}_{2}^{1}(A, B),\end{cases}
$$


гдe

$$
\Psi=\min _{t \in W_{2}^{1}\left(t^{*}, A, B\right)}\left(\sum_{j=1}^{m} a_{j} b_{t(j)}-\sum_{j=1}^{m} a_{j} b_{t^{*}(j)}\right)\left(\sum_{j=1}^{m}\left|b_{t(j)}-b_{t^{*}(j)}\right|\right)^{-1} .
$$

Следствие 6. Пусть $t^{*} \in \dot{T}_{k}^{n}(A, B), k \in N_{3}$. Если при добавлении $х$ векторному критерию $f(t, A, B)$ нового частного критерия $f_{n+1}\left(t, A_{n+1}, B_{n+1}\right)$ соответствующая эффективность подстановки $t^{*}$ сохраняется, то радиус устойчивости этой подстановки не может уменьииться.

Заметим, что при добавлении нового критерия соответствующая эффективность подстановки $t^{*} \in \dot{T}_{k}^{n}(A, B)$ может нарушиться лишь в случае, когда

$$
t^{*} \in \dot{T}_{2}^{n}(A, B) \backslash T_{1}^{n}(A, B) .
$$

Подстановку назовем устойчивой, если ее радиус устойчивости положителен.

Следствие 7. Любая строго эффективная подстановка устойчива.

Пусть $k=2,3$. Подстановку $t \in \dot{T}_{k}^{n}(A, B)$ будем называть $s$-эффективной, если

$$
\forall t^{\prime} \in W_{k}^{n}(t, A, B) \exists i \in I^{n}\left(t, t^{\prime}, B\right)\left(q_{i}\left(t, t^{\prime}, A_{i}, B_{i}\right)<0\right) .
$$

Следствие 8. Для того чтобы нетривиальная истинно (слабо) эффективнал подстановка была устойчивой, необходимо и достаточно, чтобъ она была s-эфбективной.

Поэтому оптимальная подстановка $t^{*}$ скалярной задачи $Z_{2}^{1}(A, B)$ устойчива тогда и только тогда, когда выполняется одно из условий:

$$
\begin{gathered}
T_{2}^{1}(A, B)=\left\{t^{*}\right\}, \\
\forall t \in T_{2}^{1}(A, B) \backslash\left\{t^{*}\right\}\left(\delta_{1}\left(t^{*}, t, B\right)=0\right) .
\end{gathered}
$$

По аналогии с [7-11] для любых индексов $k \in N_{3}$ и $n \in \mathrm{N}$ задачу $Z_{k}^{n}(A, B)$ назовем квазиустойчивой, если

$$
\exists \varepsilon>0 \forall A^{\prime} \in \mathscr{A}(\varepsilon)\left(T_{k}^{n}(A, B) \subseteq T_{k}^{n}\left(A+A^{\prime}, B\right)\right) .
$$

Иначе говоря, задача $Z_{k}^{n}(A, B)$ квазиустойчива, если каждая подстановка из множества $T_{k}^{n}(A, B)$ устойчива.

Отметим, что свойство квазиустойчивости задачи $Z_{k}^{n}(A, B)$ является дискретным аналогом известного (см., например, [12-14]) свойства полунепрерывности снизу (по Хаусдорфу) в точке $(A, B)$ точечно-множественного отображения

$$
T_{k}^{n}: \mathbf{R}^{n \times m} \times \mathbf{R}^{n \times m} \rightarrow 2^{T}
$$

которое каждому набору параметров векторного критерия $f(t, A, B)$ ставит в соответствие множество $T_{k}^{n}(A, B)$.

Задачу $Z_{k}^{n}(A, B)$ назовем тривиальной, если любая эффективная подстановка $t \in T_{k}^{n}(A, B)$ тривиальна. В противном случае $\left(\dot{T}_{k}^{n}(A, B) \neq \varnothing\right)$ задачу $Z_{k}^{n}(A, B)$ назовем нетривиальной. Очевидно, что всякая тривиальная задача $Z_{k}^{n}(A, B), k \in N_{3}$, квазиустойчива.

Из следствий 6 и 7 соответственно получаем следующие два утверждения. 
Следствие 9. Всяхая задача $Z_{1}^{n}(A, B), n \in \mathrm{N}$, хвазиустойчива.

Множество всех $s$-эффективных подстановок из $\dot{T}_{k}^{n}(A, B)$ обозначим $\ddot{T}_{k}^{n}(A, B)$.

Следствие 10. Пусть $k \in\{2,3\}$. Дія того чтобы нетривиалъная задача $Z_{k}^{n}(A, B)$, $n \in \mathrm{N}$, поиска множества $T_{k}^{n}(A, B)$ была квазиустойчивой, необходимо и достаточно, чтобы выполнялось равенство

$$
\dot{T}_{k}^{n}(A, B)=\ddot{T}_{k}^{n}(A, B) .
$$

Другими словами, нетривиальная задача $Z_{2}^{n}(A, B)\left(Z_{3}^{n}(A, B)\right), n \in \mathbf{N}$, квазиустойчива тогда и только тогда, когда каждая оптимальная по Парето (по Слейтеру) подстановка является $s$-эффективной.

Поэтому скалярная задача $Z_{2}^{1}(A, B)$ квазиустойчива лишь в двух случаях:

$$
\begin{aligned}
\left|T_{2}^{1}(A, B)\right| & =1 \\
\forall t \in T_{2}^{1}(A, B) \forall t^{\prime} & \in T_{2}^{1}(A, B) \backslash\{t\}\left(\delta_{1}\left(t, t^{\prime}, B\right)=0\right) .
\end{aligned}
$$

Радиусом квазиустойчивости задачи $Z_{k}^{n}(A, B), k \in N_{3}$, называется величина [7-9]

$$
\theta_{k}^{n}(A, B)= \begin{cases}\sup \Theta, & \text { если } \Theta \neq \varnothing, \\ 0 & \text { в противном случае }\end{cases}
$$

где $\Theta=\left\{\varepsilon>0: \forall A^{\prime} \in \mathscr{A}(\varepsilon)\left(T_{k}^{n}(A, B) \subseteq T_{k}^{n}\left(A+A^{\prime}, B\right)\right)\right\}$.

Поскольку

$$
\theta_{k}^{n}(A, B)=\min \left\{\rho_{k}^{n}(t, A, B): t \in T_{k}^{n}(A, B)\right\},
$$

из теоремы вытекает также следующее утверждение.

Следствие 11. При любых $n \in \mathbf{N} u k \in N_{3}$ для радиуса квазиустойчивости нетривиалъной задачи $Z_{k}^{n}(A, B)$ справедлива формула

$$
\theta_{k}^{n}(A, B)=\min _{t \in \dot{T}_{k}^{n}(A, B)} \min _{t^{\prime} \in W_{k}^{n}(t, A, B)} \max _{i \in I^{n}\left(t, t^{\prime}, B\right)} \Gamma_{i}\left(t, t^{\prime}, A_{i}, B_{i}\right) .
$$

Очевидно, что радиус квазиустойчивости всякой тривиальной задачи равен бесконечности.

Из следствия 11, в частности, получаем следующий известный результат [15].

Следствие 12. Пусть $n \in \mathrm{N},|T|>1$ и в каждой строхе матрищъ $B$ все элементы попарно различнъ. Тогда для радиуса квазиустойчивости задачи $Z_{2}^{n}(A, B)$ поиска множества Парето $T_{2}^{n}(A, B)$ справедлива формула

$$
\theta_{2}^{n}(A, B)=\min _{t \in T_{2}^{n}(A, B)} \min _{t^{\prime} \in T \backslash\{t\}} \max _{i \in N_{n}} \Gamma_{i}\left(t, t^{\prime}, A_{i}, B_{i}\right) .
$$

\section{4. Примеры}

Пример 1. Пусть

$$
A=\left(\begin{array}{ccc}
4 & -4 & -2 \\
6 & 4 & 2
\end{array}\right), \quad B=\left(\begin{array}{ccc}
0 & 1 & -3 \\
3 & 1 & -1
\end{array}\right), \quad T=\left\{t_{1}, t_{2}, t_{3}, t_{4}\right\}
$$


где

$$
t_{1}=\left(\begin{array}{lll}
1 & 2 & 3 \\
1 & 2 & 3
\end{array}\right), \quad t_{2}=\left(\begin{array}{lll}
1 & 2 & 3 \\
1 & 3 & 2
\end{array}\right), \quad t_{3}=\left(\begin{array}{lll}
1 & 2 & 3 \\
2 & 1 & 3
\end{array}\right), \quad t_{4}=\left(\begin{array}{lll}
1 & 2 & 3 \\
2 & 3 & 1
\end{array}\right)
$$

Тогда

$$
\begin{gathered}
f\left(t_{1}, A, B\right)=(2,20), \quad f\left(t_{2}, A, B\right)=f\left(t_{3}, A, B\right)=(10,16), \quad f\left(t_{4}, A, B\right)=(16,8), \\
T_{1}^{2}(A, B)=\left\{t_{1}, t_{4}\right\}, \quad T_{2}^{2}(A, B)=T_{3}^{2}(A, B)=T, \\
I^{2}\left(t_{1}, t_{2}, B\right)=I^{2}\left(t_{1}, t_{3}, B\right)=I^{2}\left(t_{2}, t_{3}, B\right)=\{1,2\}=N_{2}, \\
W_{1}^{2}\left(t_{1}, A, B\right)=W_{2}^{2}\left(t_{1}, A, B\right)=W_{3}^{2}\left(t_{1}, A, B\right)=\left\{t_{2}, t_{3}, t_{4}\right\} \\
W_{1}^{2}\left(t_{4}, A, B\right)=W_{2}^{2}\left(t_{4}, A, B\right)=W_{3}^{2}\left(t_{4}, A, B\right)=\left\{t_{1}, t_{2}, t_{3}\right\} \\
W_{2}^{2}\left(t_{2}, A, B\right)=W_{3}^{2}\left(t_{2}, A, B\right)=W_{2}^{2}\left(t_{3}, A, B\right)=W_{3}^{2}\left(t_{3}, A, B\right)=\left\{t_{1}, t_{4}\right\} .
\end{gathered}
$$

Следовательно, все подстановки множеств $T_{k}^{2}(A, B), k \in N_{3}$, нетривиальны, то есть

$$
\dot{T}_{k}^{2}(A, B)=T_{k}^{2}(A, B), \quad k \in N_{3}
$$

Легко убедиться, что

$$
\begin{aligned}
& \rho_{k}^{2}\left(t_{1}, A, B\right)=\rho_{k}^{2}\left(t_{4}, A, B\right)=1, \quad k \in N_{3}, \\
& \rho_{2}^{2}\left(t_{2}, A, B\right)=\rho_{2}^{2}\left(t_{3}, A, B\right)=\rho_{3}^{2}\left(t_{2}, A, B\right)=\rho_{3}^{2}\left(t_{3}, A, B\right)=0 .
\end{aligned}
$$

Поэтому

$$
\theta_{1}^{2}(A, B)=1, \quad \theta_{2}^{2}(A, B)=\theta_{3}^{2}(A, B)=0,
$$

то есть задачи $Z_{2}^{2}(A, B)$ и $Z_{3}^{2}(A, B)$ не являются квазиустойчивыми, а задача $Z_{1}^{2}(A, B)$, как всегда, квазиустойчива.

Пример 2. Пусть

$$
A=\left(\begin{array}{ccc}
2 & 4 & 6 \\
8 & 10 & 12
\end{array}\right), \quad B=\left(\begin{array}{ccc}
2 & 2 & 1 \\
1 & 1 & -1
\end{array}\right), \quad T=\left\{t_{1}, t_{2}, t_{3}\right\}
$$

где подстановки $t_{1}, t_{2}, t_{3}$ такие же, как в примере 1.

Тогда

$$
f\left(t_{1}, A, B\right)=f\left(t_{3}, A, B\right)=(18,6), \quad f\left(t_{2}, A, B\right)=(20,10) .
$$

Поэтому

$$
\begin{gathered}
T_{1}^{2}(A, B)=\varnothing, \quad T_{2}^{2}(A, B)=T_{3}^{2}(A, B)=\left\{t_{1}, t_{3}\right\} \\
I^{2}\left(t_{1}, t_{2}, B\right)=I^{2}\left(t_{2}, t_{3}, B\right)=N_{2}, \quad I^{2}\left(t_{1}, t_{3}, B\right)=\varnothing \\
W_{2}^{2}\left(t_{1}, A, B\right)=W_{2}^{2}\left(t_{3}, A, B\right)=W_{3}^{2}\left(t_{1}, A, B\right)=W_{3}^{2}\left(t_{3}, A, B\right)=\left\{t_{2}\right\} .
\end{gathered}
$$

Следовательно, никакая подстановка множеств $T_{2}^{2}(A, B)$ и $T_{3}^{2}(A, B)$ не является тривиальной, то есть

$$
\dot{T}_{2}^{2}(A, B)=T_{2}^{2}(A, B), \quad \dot{T}_{3}^{2}(A, B)=T_{3}^{2}(A, B) .
$$

После несложных вычислений получаем, что

$$
\rho_{2}^{2}\left(t_{1}, A, B\right)=\rho_{2}^{2}\left(t_{3}, A, B\right)=\rho_{3}^{2}\left(t_{1}, A, B\right)=\rho_{3}^{2}\left(t_{3}, A, B\right)=\theta_{2}^{2}(A, B)=\theta_{3}^{2}(A, B)=1,
$$

то есть задачи $Z_{2}^{2}(A, B)$ и $Z_{3}^{2}(A, B)$ квазиустойчивы. 
Пример 3. Пусть

$$
A=\left(\begin{array}{cccc}
1 & 2 & 3 & 1 \\
1 & -1 & -1 & 2
\end{array}\right), \quad B=\left(\begin{array}{llll}
3 & 2 & 2 & 1 \\
4 & 1 & 2 & 4
\end{array}\right), \quad T=\left\{t_{1}, t_{2}, t_{3}\right\}
$$

где

$$
t_{1}=\left(\begin{array}{llll}
1 & 2 & 3 & 4 \\
1 & 2 & 3 & 4
\end{array}\right), \quad t_{2}=\left(\begin{array}{llll}
1 & 2 & 3 & 4 \\
1 & 3 & 2 & 4
\end{array}\right), \quad t_{3}=\left(\begin{array}{llll}
1 & 2 & 3 & 4 \\
4 & 2 & 3 & 1
\end{array}\right) .
$$

Тогда $f\left(t_{k}, A, B\right)=(14,9), k \in N_{3}$. Поэтому

$$
\begin{gathered}
T_{1}^{2}(A, B)=\varnothing, \quad T_{2}^{2}(A, B)=T_{3}^{2}(A, B)=T \\
I^{2}\left(t_{1}, t_{2}, B\right)=\{2\}, \quad I^{2}\left(t_{1}, t_{3}, B\right)=\{1\}, \quad I^{2}\left(t_{2}, t_{3}, B\right)=N_{2} .
\end{gathered}
$$

При $t_{k} \in T_{2}^{2}(A, B), k \in N_{3}$, получаем, что

$$
W_{2}^{2}\left(t_{1}, A, B\right)=\left\{t_{2}, t_{3}\right\}, \quad W_{2}^{2}\left(t_{2}, A, B\right)=\left\{t_{1}, t_{3}\right\}, \quad W_{2}^{2}\left(t_{3}, A, B\right)=\left\{t_{1}, t_{2}\right\} .
$$

Таким образом, $\dot{T}_{2}^{2}(A, B)=T_{2}^{2}(A, B)$.

При $t_{k} \in T_{3}^{2}(A, B), k \in N_{3}$, находим, что

$$
W_{3}^{2}\left(t_{1}, A, B\right)=\varnothing, \quad W_{3}^{2}\left(t_{2}, A, B\right)=\left\{t_{3}\right\}, \quad W_{3}^{2}\left(t_{3}, A, B\right)=\left\{t_{2}\right\} .
$$

В результате получаем, что

$$
\dot{T}_{3}^{2}(A, B)=\left\{t_{2}, t_{3}\right\} \neq T_{3}^{2}(A, B)=\left\{t_{1}, t_{2}, t_{3}\right\},
$$

то есть подстановка $t_{1}$ тривиальна.

Далее, находим, что

$$
\begin{aligned}
\rho_{2}^{2}\left(t_{k}, A, B\right) & =0, \quad k \in N_{3}, \\
\rho_{3}^{2}\left(t_{1}, A, B\right) & =\infty, \\
\rho_{3}^{2}\left(t_{2}, A, B\right) & =\rho_{3}^{2}\left(t_{3}, A, B\right)=0, \\
\theta_{2}^{2}(A, B) & =\theta_{3}^{2}(A, B)=0,
\end{aligned}
$$

то есть задачи $Z_{2}^{2}(A, B)$ и $Z_{3}^{2}(A, B)$ не являются квазиустойчивыми.

Пример 4. Пусть

$$
A=\left(\begin{array}{cccc}
1 & 2 & -3 & 3 \\
4 & 3 & 2 & 0
\end{array}\right), \quad B=\left(\begin{array}{cccc}
2 & 2 & 1 & -1 \\
1 & 2 & 3 & 4
\end{array}\right), \quad T=\left\{t_{1}, t_{2}, t_{3}\right\}
$$

где

$$
t_{1}=\left(\begin{array}{llll}
1 & 2 & 3 & 4 \\
1 & 2 & 3 & 4
\end{array}\right), \quad t_{2}=\left(\begin{array}{llll}
1 & 2 & 3 & 4 \\
2 & 1 & 3 & 4
\end{array}\right), \quad t_{3}=\left(\begin{array}{llll}
1 & 2 & 3 & 4 \\
1 & 2 & 4 & 3
\end{array}\right)
$$

Тогда

$$
\begin{gathered}
f\left(t_{1}, A, B\right)=(0,16), \quad f\left(t_{2}, A, B\right)=(0,17), \quad f\left(t_{3}, A, B\right)=(12,18), \\
T_{1}^{2}(A, B)=T_{2}^{2}(A, B)=\left\{t_{1}\right\}, \quad T_{3}^{2}(A, B)=\left\{t_{1}, t_{2}\right\} \\
I^{2}\left(t_{1}, t_{2}, B\right)=\{2\}, \quad I^{2}\left(t_{1}, t_{3}, B\right)=I^{2}\left(t_{2}, t_{3}, B\right)=N_{2}, \\
W_{1}^{2}\left(t_{1}, A, B\right)=W_{2}^{2}\left(t_{1}, A, B\right)=\left\{t_{2}, t_{3}\right\}, \quad W_{3}^{2}\left(t_{1}, A, B\right)=\left\{t_{3}\right\}, \quad W_{3}^{2}\left(t_{2}, A, B\right)=\left\{t_{3}\right\} .
\end{gathered}
$$


Поэтому $\dot{T}_{k}^{2}(A, B)=T_{k}^{2}(A, B), k \in N_{3}$.

Далее,

$$
\begin{aligned}
\rho_{1}^{2}\left(t_{1}, A, B\right) & =\rho_{2}^{2}\left(t_{1}, A, B\right)=\theta_{1}^{2}(A, B)=\theta_{2}^{2}(A, B)=1 / 2<3 \\
& =\rho_{3}^{2}\left(t_{1}, A, B\right)=\rho_{3}^{2}\left(t_{2}, A, B\right)=\theta_{3}^{2}(A, B) .
\end{aligned}
$$

Тем самым, все задачи $Z_{k}^{2}(A, B), k \in N_{3}$, квазиустойчивы.

\section{Список литературы}

1. Подиновский В. В., Ногин В. Д., Парето-оптимальнъе решения многохритериалъных задач. Наука, Москва, 1982.

2. Танаев В. С., Шкурба В. В., Введение в теория расписаний. Наука, Москва, 1975.

3. Танаев В. С., Гордон В. С., Шафранский Я. М., Теория расписаний. Одностадийнъе системъ. Наука, Москва, 1984.

4. Емеличев В. А., Супруненко Д. А., Танаев В. С., О работе белорусских математиков в области дискретной оптимизации. Известия $A H$ CCCP. Техническая кибернетиха (1982) №6, 25-45.

5. Леонтьев В. К., Устойчивость задачи коммивояжера. Журнал вычисл. матем. и матем. физики (1975) 15, №5, 1293-1309.

6. Леонтьев В. К., Устойчивость в линейных дискретных задачах. Пробл. кибернетихи. (1979) 35, 169-184.

7. Емеличев В. А., Кравцов М. К., Об устойчивости в траекторных задачах векторной оптимизации. Кибернетиха и системнъй анализ. (1995) №4, 137-143.

8. Емеличев В. А., Кравцов М. К., Подкопаев Д. П., О квазиустойчивости траекторных задач векторной оптимизации. Матем. заметки. (1998) 63, №1, 21-27.

9. Емеличев В. А., Подкопаев Д. П., О количественной мере устойчивости векторной задачи целочисленного программирования. Журнал вычисл. матем. и матем. физики. (1998) 38, №11, 1801-1805.

10. Бердышева Р. А., Емеличев В. А., Некоторые виды устойчивости комбинаторной задачи лексикографической оптимизации. Изв. высших учебнъх заведений. Математика. (1998) №12, 11-21.

11. Емеличев В. А., Бердышева Р. А., О радиусах устойчивости, квазиустойчивости и стабильности векторной траекторной задачи лексикографической оптимизации. Дискретнал математиха (1998) 10, №1, 20-27.

12. Молодцов Д. А., Устойчивость принципов оптимальности. Наука, Москва, 1987.

13. Белоусов Е. Г., Андронов В. Г., Разрешимость и устойчивость задач полиномиального программирования. Изд. МГУ, Москва, 1993.

14. Сергиенко И. В., Козерацкая Л. Н., Лебедева Т. Т., Исследование устойчивости и параметрический анализ дискретных оптимизачионяых задач. Наукова думка, Киев, 1995.

15. Емеличев В. А., Похилько В. Г., Радиус квазиустойчивости множества Парето векторной задачи минимизации линейных форм на множестве подстановок. Вестних Белорус. yя-ma, Cep. 1. (1999) N3, 45-47. 